\title{
A FURTHER NOTE ON THE CONVERSE OF FERMAT'S THEOREM
}

BY D. H. LEHMER

In a previous paper* the writer had discussed the converse of Fermat's theorem as a means of establishing the primality or non-primality of a large integer. Use was made chiefly of the following theorem:

Theorem 3. If $a^{x} \equiv 1(\bmod N)$ for $x=N-1$ and if $a^{x} \equiv r \neq 1$ for $x=(N-1) / p$ and if $r-1$ is prime to $N$, then all the factors of $N$ belong to the form $n p^{\alpha}+1$ where $\alpha$ is the highest power of the prime $p$ contained in $N-1$.

It is the purpose of this note to give a more general theorem in which the third part of the hypothesis of Theorem 3 is removed.

THEOREM 4. If $a^{x} \equiv 1(\bmod N)$ for $x=N-1$ and $a^{x} \equiv r \not \equiv 1$ for $x=(N-1) / p$, then all the factors of $N / \delta$ are of the form $n p^{\alpha}+1$, where $\alpha$ is the highest power of the prime $p$ contained in $N-1$ and where $\delta$ is the G.C.D. of $r-1$ and $N$.

Let $k$ be a prime factor of $N / \delta$ and let $\omega$ be the exponent to which $a$ belongs modulo $k$. Then $\omega$ divides $N-1$ and $k-1$ but not $m=(N-1) / p$; for if $\omega$ divided $m$ we would have $a^{m} \equiv 1(\bmod k)$ so that $r-1$ would divide by $k$. But this is impossible, since $k$ divides $N / \delta$ which is prime to $r-1$. From here on, the proof is the same as in Theorem 3 with the result that $k=n p^{\alpha}+1$.

Ordinarily, we have $\delta=1$ so that the two theorems become identical. An example in which this is not the case is the following: Let $N=16,046,641 . \quad N-1=2^{4} \times 3^{3} \times 5 \times 17 \times 19$ $\times 23$. It will be found that

\footnotetext{
* This Bulletin, vol. 33 (1927), pp. 327-340.
} 


$$
\begin{aligned}
2^{N-1} & \equiv 1(\bmod N), \\
2^{(N-1) / 19} & =8708025=r .
\end{aligned}
$$

If we take the G.C.D. of $r-1$ and $N$, we get $\delta=35113$, so that $N / \delta=457$ and we are able to say that the factors of 457 are of the form $19 n+1$. The factors of $\delta$ may be obtained by applying Theorem 4 in which a new value of $m$ is chosen. The modulus may be taken as $\delta$.

Theorem 4 has been applied to the number

$$
N=\left(2^{61}+1\right) / 3=768614336404564651 .
$$

This number has been listed as a prime but the writer could not find any account of its investigation.*

The factorization of $N-1$ is

$$
\begin{aligned}
N-1=2\left(2^{60}-1\right) / 3= & 2 \cdot 3 \cdot 5^{2} \cdot 7 \cdot 11 \cdot 13 \cdot 31 \cdot 41 \cdot 61 \\
& \cdot 151 \cdot 331 \cdot 1321 .
\end{aligned}
$$

It was found that

$$
\begin{aligned}
3^{(N-1) / 5} & \equiv 754529885435533861=r_{1}, \\
3^{(N-1) / 3} & \equiv 243065045915817725=r_{2}, \\
3^{N-1} & \equiv 1 .
\end{aligned}
$$

It happens that $r_{1}-1$ and $r_{2}-1$ are both prime to $N$. Hence the factors of $N$ belong to the forms

$$
\left.\begin{array}{r}
3 n+1 \\
25 n+1 \\
61 n+1
\end{array}\right\} 4575 n+1
$$

By making use of the fact that $N$ has no factor $<300,000$ and by seeking to represent $N$ as the difference of two squares it was easily shown to be a prime.

Two more numbers dividing $10^{n} \pm 1$ have also been tested recently. The first of these is $\left(10^{37}-1\right) / 9$ or

$$
N=1,111,111,111,111,111,111,111,111,111,111,111,111 \text {. }
$$

* Cunningham and Woodall, Factorisation of $y^{n} \pm 1$, London, 1925, p. 1. 
It was found that

$7^{N-1} \equiv 618,117,398,624,349,204,361,513,620,865,505,749$

$(\bmod N)$.

Hence $N$ is composite. This number furnishes another example of the scarcity of primes of this form. The next such number which has any chance of primality consists of 47 of the digits 1 .

The second number tested is $\left(10^{41}+1\right) / 11$ or

$N=$

9,090,909,090,909,090,909,090,909,090,909,090,909,091.

In this case it was found that

$3^{N-1} \equiv$

$763,287,007,500,473,474,161,903,784,495,157,879,509$

$(\bmod N)$.

It follows, then, that $N$ is also composite. This result represents the sixth attempt and failure to discover a larger prime than $2^{127}-1$ found by Lucas in 1877 .

The University of California

\section{ON THE APPROXIMATE REPRESENTATION OF ANALYTIC FUNCTIONS*}

\section{BY DUNHAM JACKSON}

The purpose of this paper is to discuss the convergence of approximating polynomials determined by a least-square criterion, together with certain auxiliary conditions. Let $f(x)$ be a given function over the interval $a \leqq x \leqq b$. For each positive integral value of $n$, let $p_{n}$ be a positive integer $\leqq n$. A polynomial of the $n$th degree may be required, for example, to coincide in value with $f(x)$ at $p_{n}$ specified points of the interval; and among the infinitely many polynomials of the

* Presented to the Society, September 8, 1927. 